\title{
Performance Degradation of Phase-Modulated Systems due to Nonlinear Phase Noise
}

\author{
Keang-Po Ho, Member, IEEE
}

\begin{abstract}
The error probability is calculated for phase-modulated systems with nonlinear phase noise. Using the assumption that the phase of amplifier noise and nonlinear phase noise are independent of each other, the error probability and penalty are calculated for both phase-shift keying (PSK) and differential phaseshift keying (DPSK) systems. The mean nonlinear phase shift must be less than about 1.00 and 0.63 rad for a penalty less than $1 \mathrm{~dB}$ for PSK and DPSK systems, respectively.
\end{abstract}

Index Terms-Fiber nonlinearities, nonlinear phase noise, phase modulation.

\section{INTRODUCTION}

W HEN OPTICAL amplifiers are used to compensate for fiber loss, the interaction of fiber Kerr effect and optical amplifier noise generates nonlinear phase noise, often called the Gordon-Mollenauer effect [1]. Nonlinear phase noise affects both phase-shift keying (PSK) and differential phase-shift keying (DPSK) systems [2]-[4] that have renewed interests recently [5]-[7]. Recently, Kim and Gnauck [4] show experimentally and the author [8], [9] shows theoretically that the nonlinear phase noise is not Gaussian distributed and variance or $Q$ factor [1], [3] is not sufficient to characterize the system performance. Using the probability density function (pdf) of nonlinear phase noise derived in [9], this letter investigates the performance of phase-modulated systems with nonlinear phase noise.

Based on the assumption that the nonlinear phase noise is independent of the phase component of the received electric field without nonlinear phase noise, the error probability is calculated for both PSK and DPSK systems. The signal-to-noise (SNR) penalty is also evaluated for an error probability of $10^{-9}$. Simulation is conducted to verify the theoretical calculation.

\section{ERror Probability With Nonlinear Phase Noise}

A PSK signal can be demodulated using an optical phaselocked loop [10]. Linear phase noise induced by an optical amplifier, called the phase of amplifier noise of $\Theta_{n}$ here, has a pdf of [11, Sec. 5.2.7], [12]

$$
\begin{aligned}
p_{\Theta_{n}}(\theta)=\frac{1}{2 \pi} e^{-\rho_{s}} & \\
& +\sqrt{\frac{\rho_{s}}{4 \pi}} \cos \theta e^{-\rho_{s} \sin ^{2} \theta} \operatorname{erfc}\left(-\sqrt{\rho_{s}} \cos \theta\right)
\end{aligned}
$$

\footnotetext{
Manuscript received February 6, 2003; revised May 8, 2003

The author is with the Graduate Institute of Communication Engineering, National Taiwan University, Taipei 106, Taiwan, R.O.C. (e-mail: kpho@cc.ee.ntu.edu.tw).

Digital Object Identifier 10.1109/LPT.2003.816704
}

where $\rho_{s}$ is the $\operatorname{SNR}$ and $\operatorname{erfc}(\cdot)$ is the complementary error function. The characteristic function of the phase of amplifier noise is

$$
\Psi_{\Theta_{n}}(j \nu)=\int_{-\pi}^{+\pi} p_{\Theta_{n}}(\theta) e^{-j \nu \theta} d \theta
$$

Because the pdf of $p_{\Theta_{n}}(\theta)$ is an even function, the characteristic function of $\Psi_{\Theta_{n}}(j \nu)$ is a real function. The characteristic function of $\Psi_{\Theta_{n}}(j \nu)$ can be found in [13].

When the number of fiber spans is very large, the normalized nonlinear phase noise of $\Phi$ has a characteristic function of [9]

$$
\Psi_{\Phi}(j \nu)=\sec (\sqrt{j \nu}) \exp \left[\rho_{s} \sqrt{j \nu} \tan (\sqrt{j \nu})\right] .
$$

With nonlinear phase noise, if the transmitted phase is zero, the overall received phase is

$$
\Phi_{r}=\Theta_{n}-\Phi_{\mathrm{NL}}=\Theta_{n}-\frac{\left\langle\Phi_{\mathrm{NL}}\right\rangle}{\rho_{s}+\frac{1}{2}} \Phi
$$

where $\Phi_{\mathrm{NL}}$ is the nonlinear phase noise, $\left\langle\Phi_{\mathrm{NL}}\right\rangle$ is the mean nonlinear phase shift, and $\langle\Phi\rangle=\rho_{s}+1 / 2$ is the mean normalized phase. If the phase of amplifier noise of $\Theta_{n}$ is independent of the nonlinear phase noise of $\Phi_{\mathrm{NL}}$, the characteristic function of the received phase of $\Phi_{r}$ is

$$
\Psi_{\Phi_{r}}(j \nu)=\Psi_{\Theta_{n}}(j \nu) \Psi_{\Phi}\left(-j \nu \frac{\left\langle\Phi_{\mathrm{NL}}\right\rangle}{\rho_{s}+\frac{1}{2}}\right) .
$$

The pdf of the received phase is the inverse Fourier transform of the above PSK characteristic function

$$
p_{\Phi_{r}}(\phi)=\frac{1}{2 \pi} \int_{-\infty}^{+\infty} \Psi_{\Phi_{r}}(j \nu) e^{j \phi \nu} d \nu
$$

and the error probability is

$$
p_{e, \mathrm{PSK}}=1-\int_{-\pi / 2-\left\langle\Phi_{\mathrm{NL}}\right\rangle}^{\pi / 2-\left\langle\Phi_{\mathrm{NL}}\right\rangle} p_{\Phi_{r}}(\phi) d \phi
$$

where the decision boundary of $\pm \pi / 2$ is rotated by the mean nonlinear phase shift of $\left\langle\Phi_{\mathrm{NL}}\right\rangle$.

A DPSK system is demodulated using interferometer [4]-[7] to deduce the differential phase of

$$
\begin{aligned}
\Delta \Phi_{r} & =\Phi_{r}(t)-\Phi_{r}(t-T) \\
& =\Theta_{n}(t)-\Phi_{\mathrm{NL}}(t)-\Theta_{n}(t-T)+\Phi_{\mathrm{NL}}(t-T)
\end{aligned}
$$

where $\Phi_{r}(\cdot), \Theta_{n}(\cdot)$, and $\Phi_{\mathrm{NL}}(\cdot)$ are the received phase, the phase of amplifier noise, and the nonlinear phase noise as a function of time and $T$ is the symbol interval. The phases at $t$ and $t-T$ are independent of each other but are identically distributed 


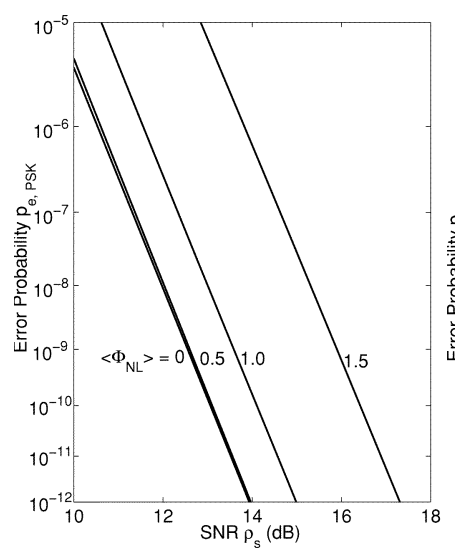

(a)

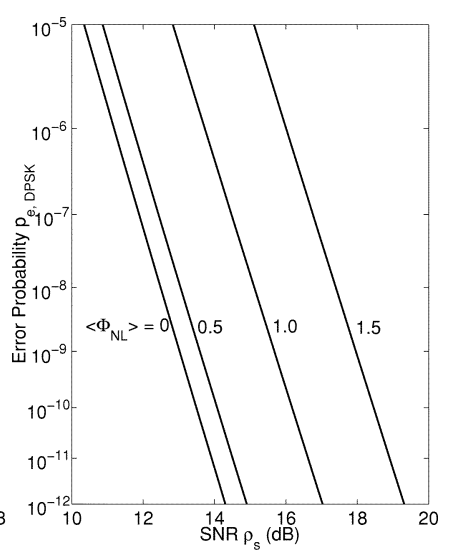

(b)
Fig. 1. Error probability as a function of SNR for (a) PSK and (b) DPSK systems.

random variables similar to that of (4). The differential phase of (8) assumes that the transmitted phases at $t$ and $t-T$ are the same. The characteristic function of the differential phase is

$$
\Psi_{\Delta \Phi_{r}}(j \nu)=\Psi_{\Theta_{n}}^{2}(j \nu)\left|\Psi_{\Phi}\left(j \nu \frac{\left\langle\Phi_{\mathrm{NL}}\right\rangle}{\rho_{s}+\frac{1}{2}}\right)\right|^{2} .
$$

Taking the inverse Fourier transform of $\Psi_{\Delta \Phi_{r}}(j \nu)$ gives the pdf of the differential phase of $p_{\Delta \Phi_{r}}(\phi)$. The error probability of DPSK system is

$$
p_{e, \mathrm{DPSK}}=1-\int_{-\pi / 2}^{\pi / 2} p_{\Delta \Phi_{r}}(\phi) d \phi .
$$

Because the DPSK characteristic function of $\Psi_{\Delta \Phi_{r}}(j \nu)$ is a real function, the pdf of $p_{\Delta \Phi_{r}}(\phi)$ is an even function.

\section{NUMERICAL RESULTS}

Fig. 1 show the error probability for PSK and DPSK systems with nonlinear phase noise as a function of SNR $\rho_{s}$. Fig. 1(a) is the error probability for PSK systems calculated by (7) and Fig. 1(b) is the error probability for DPSK systems calculated by (10). Fig. 1 is calculated for a mean nonlinear phase shift of $\left\langle\Phi_{\mathrm{NL}}\right\rangle=0,0.5,1.0$, and $1.5 \mathrm{rad}$. Without nonlinear phase noise of $\left\langle\Phi_{\mathrm{NL}}\right\rangle=0$, the error probabilities are equal to $\operatorname{erfc}\left(\sqrt{\rho_{s}}\right) / 2$ [11, Sec. 5.2.7] and $\exp \left(-\rho_{s}\right) / 2$ [11, Sec. 5.2.8] for PSK and DPSK systems, respectively. From Fig. 1, DPSK systems are more sensitive to nonlinear phase noise than PSK systems.

Fig. 2 plots the required SNR for an error probability of $10^{-9}$ versus the mean nonlinear phase shift of $\left\langle\Phi_{\mathrm{NL}}\right\rangle$. The mean nonlinear phase shift must be less than about 1.00 and $0.63 \mathrm{rad}$ for a penalty less than $1 \mathrm{~dB}$ for PSK and DPSK systems, respectively. The SNR penalty is negligible for PSK and DPSK systems when the mean nonlinear phase shift of $\left\langle\Phi_{\mathrm{NL}}\right\rangle$ is less than 0.6 and $0.4 \mathrm{rad}$, respectively. The mean nonlinear phase shift must be less than about 1.19 and 0.85 rad for a penalty less than $2 \mathrm{~dB}$ for PSK and DPSK systems, respectively.

The optimal operating level is that the increase of mean nonlinear phase shift, proportional to the increase of launched power and SNR, does not decrease the system performance.

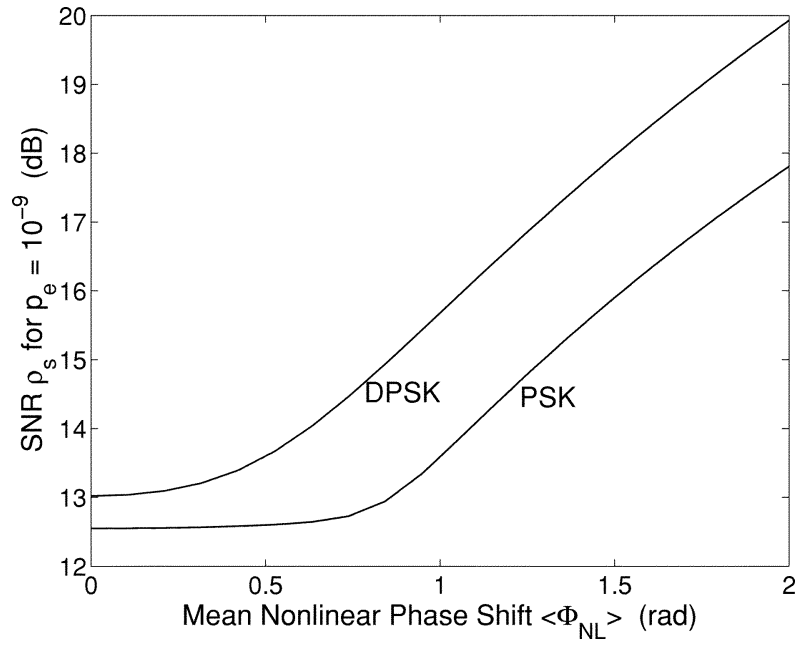

Fig. 2. Required SNR for an error probability of $10^{-9}$ as a function of the mean nonlinear phase shift of $\left\langle\Phi_{\mathrm{NL}}\right\rangle$.

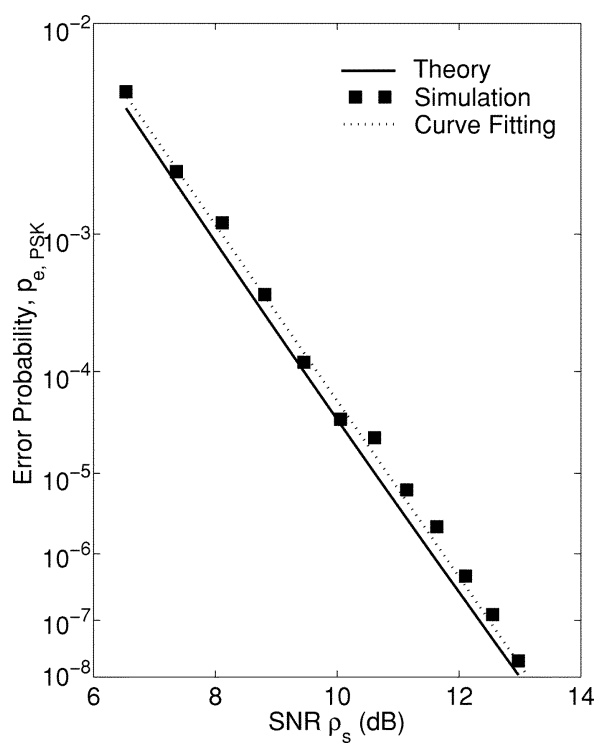

Fig. 3. Calculated and simulated error probability for a PSK system for a mean nonlinear phase shift of $\left\langle\Phi_{\mathrm{NL}}\right\rangle=1 \mathrm{rad}$.

For Fig. 2, the optimal operation point can be found by $d \rho_{s} / d\left\langle\Phi_{\mathrm{NL}}\right\rangle \rightarrow 1$ when both the required SNR $\rho_{s}$ and mean nonlinear phase shift $\left\langle\Phi_{\mathrm{NL}}\right\rangle$ are expressed in decibels. About the same as the estimation of [1], the optimal mean nonlinear phase shifts are 0.97 and 0.92 rad for PSK and DPSK signals, respectively.

To verify the accuracy of the error probability in Fig. 1(a), Fig. 3 compares the theoretical and simulated error probability as a function of SNR for a typical PSK system having $\left\langle\Phi_{\mathrm{NL}}\right\rangle=$ $1 \mathrm{rad}$ [1]. The simulation is conducted for 32 fiber spans based on Monte Carlo error counting. The distribution of the received electric field $E_{N} \exp \left(-j \Phi_{\mathrm{NL}}\right)$ is found where, depending on each other, $E_{N}$ is the electric field with only amplifier noise and $\Phi_{\mathrm{NL}}$ is given by the model of [8] for 32 fiber spans. The error count is equal to the number of points outside the decision region. Although DPSK systems have more practical applications, PSK systems are simulated for its low memory and time requirement. The theoretical results are the same as that in Fig. 1(a) but 
extend to high error probability. Fig. 3 shows that the theoretical and simulation results have an insignificant difference of about $0.15 \mathrm{~dB}$. Part of the difference is because the pdf for 32 fiber spans has slightly larger tail probability than that corresponding to (3) [9]. Nevertheless, a discrepancy within $0.15 \mathrm{~dB}$ (or 3\%) is more than sufficient to characterize a system.

\section{DISCUSSION}

The derivation in this letter uses the independent assumption that the phase of amplifier noise $\Theta_{n}$ and the nonlinear phase noise of $\Phi_{\mathrm{NL}}$ are independent of each other. Because the nonlinear phase noise of $\Phi_{\mathrm{NL}}$ is due to the accumulation of self-phase modulation that is proportional to the intensity of the signal, it is easy to show that the phase of amplifier noise $\Theta_{n}$ is uncorrelated to the nonlinear phase noise of $\Phi_{\mathrm{NL}}$, i.e., $E\left\{\Theta_{n} \Phi_{\mathrm{NL}}\right\}=0$ where $E\{\cdot\}$ denotes expectation. Although uncorrelated is equivalent to independence for two Gaussian random variables, both the phase of amplifier noise $\Theta_{n}$ and the nonlinear phase noise of $\Phi_{\mathrm{NL}}$ are close to but not exactly Gaussian random variables [4], [8], [9], [11, Sec. 5.2.7]. While the independent assumption cannot absolutely assured, with weak dependence, the calculation of Fig. 1 is very accurate, as confirmed by Fig. 3.

This letter assumes that the optical filter before the receiver is a matched filter and the electrical filter after the photodetector does not further distort the signal. Similar to [1] and [9], nonlinear phase noise is induced by self-phase modulation of the signal and amplifier noise within an optical bandwidth matched to the signal for dispersionless fiber. With the self-phase modulation assumption, both signal and noise propagate in the same speed even for dispersive fiber and the characteristic function of (3) is a very good approximation. If the nonlinear phase noise induced by cross-phase modulation is taken into account, the characteristic function of (3) may require small modification but the independent assumption between adjacent symbols to derive (9) is not valid for dispersive fiber.

Similar to [1], [8], and [9], all the derivation here assumes nonreturn-to-zero pulses but most experiments [5], [7] use the return-to-zero (RZ) pulses. For the RZ pulse, the mean nonlinear phase shift of $\left\langle\Phi_{\mathrm{NL}}\right\rangle$ in (4) should be the mean nonlinear phase shift when the peak amplitude is transmitted. Usually, $\left\langle\Phi_{\mathrm{NL}}\right\rangle$ is increased by the inverse of the duty cycle.

The tail of the pdf corresponding to the characteristic function of (3) is asymmetrical with respect to the normalized mean of $\rho_{s}+1 / 2$. The pdf of (6) is also asymmetrical with respect to the mean of $\left\langle\Phi_{\mathrm{NL}}\right\rangle$. The error probability of (7) assumes that the pdf of (6) is symmetrical with respect to $\left\langle\Phi_{\mathrm{NL}}\right\rangle$. Lower error probability than that of (7) can be achieved using an "asymmetrical" decision boundary. For the more practical DPSK systems, the pdf of $p_{\Delta \Phi_{r}}(\phi)$ is an even function; the decision boundary of $\pm \pi / 2$ is the optimal for the error probability of (10).

In practice, an interferometer-based detector [4]-[7] makes a decision on whether $\cos \left(\Delta \Phi_{r}\right)$ is positive or negative. This detection method is equivalent to whether the differential phase of $\Delta \Phi_{r}(8)$ is within or without $\pm \pi / 2$ in the integral of (10).
The error probability given by (7) and (10) is valid for small error probability. When the error probability is high and with nonlinear phase noise, as an example, a nonlinear phase noise larger than $\pm 2 \pi$ still gives a correct decision. In the extreme case of high error probability, both (7) and (10) overestimate the error probability.

The SNR $\rho_{s}$ of Figs. 1-3 is defined the same as that in [9], [12], and [13]. When optical SNR (OSNR) is measured using an optical spectrum analyzer with a bandwidth of $\mathrm{BW}_{\text {opt }}$, the SNR is related to OSNR by $\rho_{s}=2 \mathrm{OSNR} \times \mathrm{BW}_{\text {opt }} / R_{b}$ where $R_{b}$ is the data rate of the signal and the factor of two assumes a polarization-insensitive optical spectrum analyzer.

\section{CONCLUSION}

The error probability is calculated for PSK and DPSK systems using a pdf of the summation of the phase of amplifier noise and the nonlinear phase noise. The required SNR for an error probability of $10^{-9}$ is also calculated as a function of the mean nonlinear phase shift. For the typical case of having a mean nonlinear phase shift of $1 \mathrm{rad}$, simulation results confirm that the theoretical calculation is accurate within $0.15 \mathrm{~dB}$. The mean nonlinear phase shift must be less than about 1.00 and $0.63 \mathrm{rad}$ for an SNR penalty less than $1 \mathrm{~dB}$ for PSK and DPSK systems, respectively.

\section{REFERENCES}

[1] J. P. Gordon and L. F. Mollenauer, "Phase noise in photonic communications systems using linear amplifiers," Opt. Lett., vol. 15, pp. $1351-1353,1990$

[2] S. Ryu, "Signal linewidth broadening due to nonlinear Kerr effect in long-haul coherent systems using cascaded optical amplifiers," J. Lightwave Technol., vol. 10, pp. 1450-1457, Oct. 1992.

[3] C. J. McKinstrie and C. Xie, "Phase jitter in single-channel soliton system with constant dispersion," IEEE J. Select. Topics Quantum Electron., vol. 8, pp. 616-625, May/June 2002.

[4] H. Kim and A. H. Gnauck, "Experimental investigation of the performance limitation of DPSK systems due to nonlinear phase noise," IEEE Photon. Technol. Lett., vol. 15, pp. 320-322, Feb. 2003.

[5] A. H. Gnauck et al., " $2.5 \mathrm{~Tb} / \mathrm{s}(64 \times 42.7 \mathrm{~Gb} / \mathrm{s})$ transmission over $40 \times 100 \mathrm{~km}$ NZDSF using RZ-DPSK format and all-Raman-amplified spans," in Proc. OFC, San Jose, CA, 2002, Postdeadline Paper FC2.

[6] R. A. Griffin et al., " $10 \mathrm{~Gb} / \mathrm{s}$ optical differential quadrature phase shift key (DQPSK) transmission using GaAs/AlGaAs integration," in Proc. OFC, San Jose, CA, 2002, Postdeadline Paper FD6.

[7] B. Zhu et al., "Transmission of $3.2 \mathrm{~Tb} / \mathrm{s}(80 \times 42.7 \mathrm{~Gb} / \mathrm{s})$ over 5200 $\mathrm{km}$ of UltraWave ${ }^{\mathrm{TM}}$ fiber with $100-\mathrm{km}$ dispersion-managed spans using RZ-DPSK format," in Proc. ECOC, Copenhagen, Denmark, 2003, Postdeadline Paper PD4.2.

[8] K.-P. Ho, "Probability density of nonlinear phase noise," J. Opt. Soc. Amer. B, Opt. Phys., vol. 20, no. 9, Sept. 2003, to be published.

[9] — , "Asymptotic probability density of nonlinear phase noise," Opt. Lett., vol. 28, no. 15, Aug. 2003, to be published.

[10] S. Norimatsu, K. Iwashita, and K. Noguchi, "An 8-Gb/s QPSK optical homodyne detection experiment using external-cavity laser diodes," IEEE Photon. Technol. Lett., vol. 4, pp. 765-767, July 1992.

[11] J. G. Proakis, Digital Communications, 4th ed. Boston, MA: McGrawHill, 2000.

[12] R. F. Pawula, "Distribution of the phase angle between two vectors perturbed by Gaussian noise II," IEEE Trans. Veh. Technol., vol. 50, pp. 570-575, Mar. 2001.

[13] D. Middleton, An Introduction to Statistical Comunication Theory. New York: McGraw-Hill, 1960, pp. 414-419. 